\title{
Microanalysis and Piezoelectric Properties of Piezoelectric Film Sensors Based on Heat Treatment Process
}

\author{
Yudong Zhang, Jin Guo,* Yan Han, and Jing Mi \\ Hebei Key Laboratory for Diagnosis, Reconstruction and Anti-disaster of Civil Engineering \\ Zhangjiakou 075000, China
}

(Received March 19, 2018; accepted June 5, 2018)

Keywords: piezoelectric ceramic particles, sensors, heat treatment, application research

Purchased piezoelectric ceramic particles have a high surface activity, a low dielectric constant, and a low ferroelectric performance. The piezoelectric activity of piezoelectric ceramic particles was further improved by improving the interface bonding state between the piezoelectric ceramic particles and the polymer matrix. In this study, the piezoelectric ceramic particles were treated at a high temperature under nitrogen protection. Crystal forms of the piezoelectric ceramic particles were analyzed via X-ray diffraction (XRD) and scanning electron microscopy (SEM) technologies. The dielectric and piezoelectric properties of the piezoelectric film, which were influenced by the heat treatment process, were the focus of this study. The heat treatment temperature of the piezoelectric ceramic particles was determined, and piezoelectric film sensors were prepared. The applied research of the piezoelectric film sensors was performed via a cantilever beam experiment. The results showed that the heat treatment resulted in the decomposition of strong groups at the piezoelectric ceramic particle surface, the surface activity of the particles decreased, the interface compatibility of the piezoelectric ceramic particles and the polymer matrix was enhanced, the reasonable heat treatment temperature was $1200{ }^{\circ} \mathrm{C}$, and the piezoelectric film sensors that were used for the real-time monitoring of structures were effective.

\section{Introduction}

The preparation of piezoelectric ceramic particles is typically divided into four steps: collection of ingredients, presintering, molding, and high-temperature sintering. ${ }^{(1-3)}$ The block broken method is the most common method used in production, as it reduces the production cost. This method results in the surface damage of the particles, the multidomain, and the dielectric, as well a reduction in the ferroelectric properties. ${ }^{(4)}$ The surface activity of the piezoelectric ceramic particles significantly increased during the preparation process, which leads to the piezoelectric ceramic particle surface absorbing some organic impurities or the water in the air in order to produce hydroxyl groups $(-\mathrm{OH}){ }^{(5)}$ The compatibility between the piezoelectric ceramic particles and the polyvinylidene fluoride (PVDF) matrix is poor, owing to

\footnotetext{
*Corresponding author: e-mail: zhangyudong1028@163.com

** Corresponding author: e-mail: JinGuo@stdu.edu.cn

https://dx.doi.org/10.18494/SAM.2018.1943
} 
the existence of strong groups, which causes additional defects between the two phases. ${ }^{(6-8)}$

In this study, the piezoelectric ceramic particles were treated at a high temperature under nitrogen protection. The interface bonding state between the piezoelectric ceramic particles and the polymer matrix was improved. Crystal forms of the piezoelectric ceramic particles were analyzed via X-ray diffraction (XRD) and scanning electron microscopy (SEM) technologies. The dielectric and piezoelectric properties of the film, which were influenced by the heat treatment process, were the focus of this study.

\section{Materials and Methods}

\subsection{Materials}

- Functional phase: Lead zirconate titanate (PZT) ceramic particles were used for the functional phase, which were produced by Bailing Ceramic Limited Company. The molecular symbol for piezoelectric ceramic is $\mathrm{Pb}\left(\mathrm{Zr}_{0.52} \mathrm{Ti}_{0.48}\right) \mathrm{O}_{3}$.

- Matrix phase: PVDF powders were used for the matrix phase, which were produced by Shanghai 3F New Materials Co. Ltd. The major parameters of the PVDF powders are shown in Table 1.

- Conductive phase: Carbon nanotubes were used for the conductive phase, which were generated by Shenzhen Nanotech Port Co. Ltd. The major parameters of carbon nanotubes are shown in Table 2.

\subsection{Heat treatment of PZT ceramic particles}

Piezoelectric ceramic particles were layered in the corundum crucible and heat treated under nitrogen protection. The heat treatment device for PZT powders is shown in Fig. 1. The heat treatment temperatures were $600,800,1000,1100,1150,1200$, and $1250{ }^{\circ} \mathrm{C}$.

\subsection{Fabrication of piezoelectric film sensors}

The piezoelectric films (0-3 type) that were doped with carbon nanotubes were prepared by flowing. The fabrication process is shown in Fig. 2. The volume content of the PZT ceramic particles was $50 \%$. That of the carbon nanotubes was $50 \%$.

Table 1

Major parameters of PVDF powders.

\begin{tabular}{lccc}
\hline Name & Model & $\rho\left(\mathrm{Kg} / \mathrm{m}^{3}\right)$ & Melting point $\left({ }^{\circ} \mathrm{C}\right)$ \\
\hline PVDF & FR905 & 1750 & 160
\end{tabular}

Table 2

Major parameters of carbon nanotubes.

\begin{tabular}{lccc}
\hline Name & Diameter $(\mathrm{nm})$ & Specific area $\left(\mathrm{m}^{2} / \mathrm{g}\right)$ & Length $(\mu \mathrm{m})$ \\
\hline Carbon nanotubes & $10-20$ & $10-300$ & $5-15$ \\
\hline
\end{tabular}




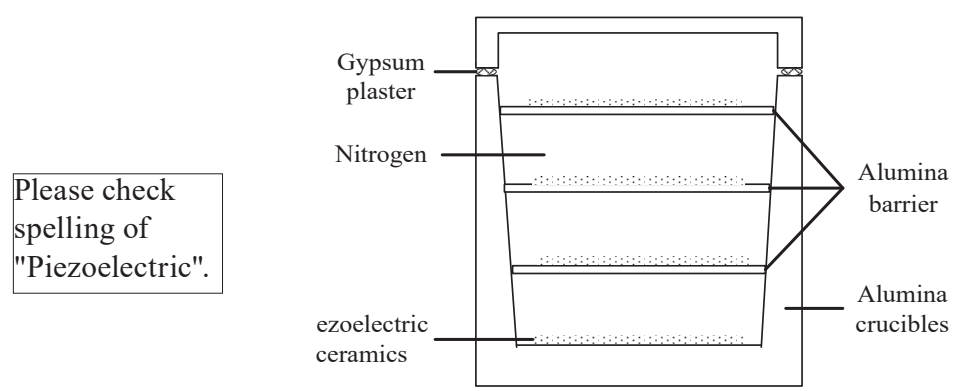

Fig. 1. Heat treatment device for PZT powders.

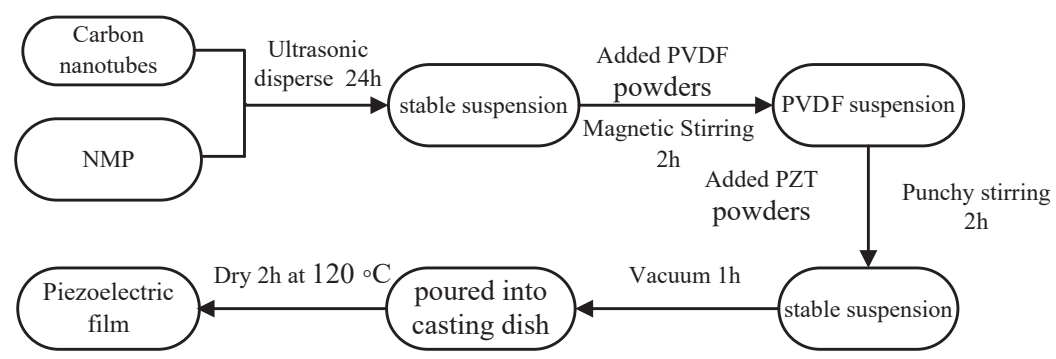

Fig. 2. Preparation of piezoelectric film.

A silver slurry was then coated on the surface of the piezoelectric film after polarization. Wires were attached to the surface of the sample. Insulation films were used to insulate the film. The piezoelectric film sensors are shown in Fig. 3.

\subsection{Cantilever beam experiment}

The piezoelectric film sensors and strain gauge were attached to the surface of the equalstrength cantilever steel beam. A vibrator was used to vibrate the beam. DSPACE was used to collect the signals from the piezoelectric film sensors and strain gauge concurrently. The experimental device is shown in Fig. 4.

\section{Analysis of Heat Treatment}

\subsection{Appearances of PZT ceramic particles}

The appearances of the PZT ceramic particles treated at various temperatures are shown in Fig. 5. The initial powders were gray. As the heat treatment temperature increased, the PZT ceramic particles became light yellow, and finally, dark yellow. When the heat treatment temperature was over $1000{ }^{\circ} \mathrm{C}$, the PZT ceramic particles began to condense with the loss of mass. When the temperature reached $1200{ }^{\circ} \mathrm{C}$, high-hardness PZT ceramic particles were formed. When the temperature reached $1250{ }^{\circ} \mathrm{C}$, massive PZT ceramic particles were formed. 


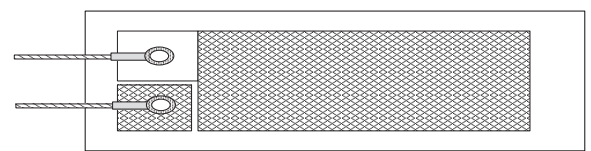

Fig. 3. Piezoelectric film sensors.

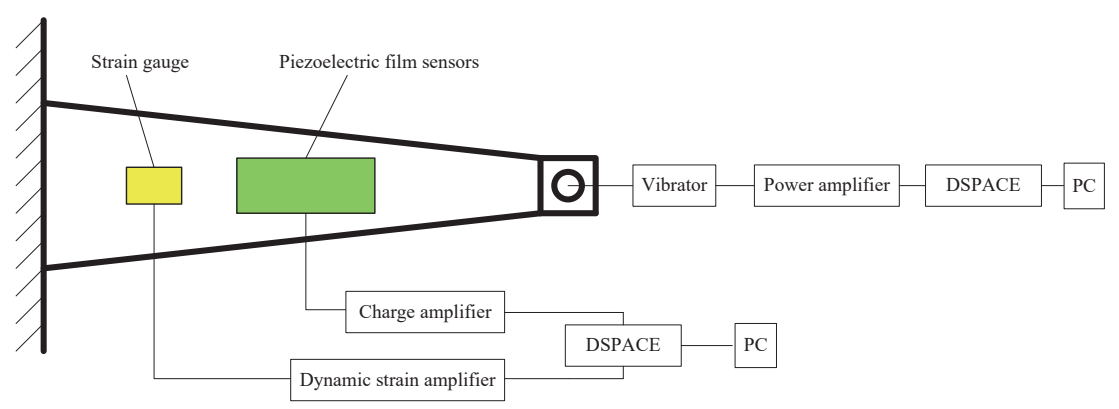

Fig. 4. (Color online) Diagram of the cantilever beam test.

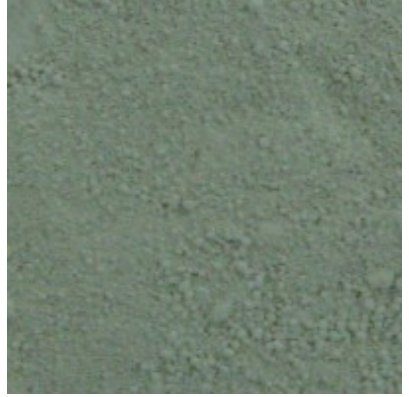

(a)

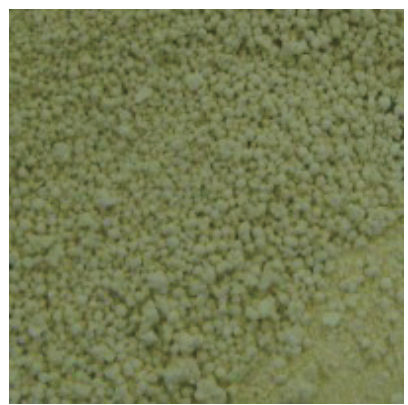

(c)

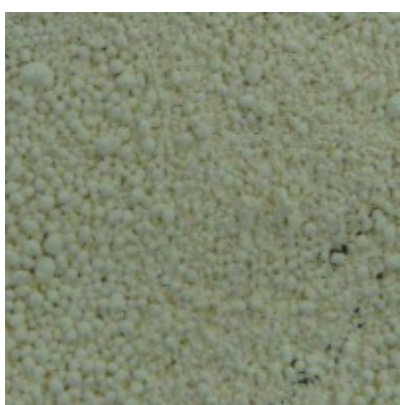

(b)

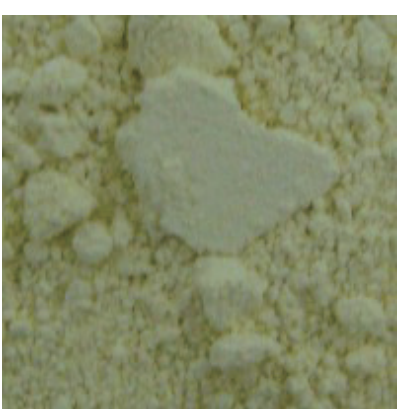

(d)

Fig. 5. (Color online) Appearances of PZT ceramic particles treated at various temperatures. (a) Initial powder, (b) 1000, (c) 1200 , and (d) $1250{ }^{\circ} \mathrm{C}$.

\subsection{XRD patterns of PZT ceramic particles}

The piezoelectric ceramic is a multiphase coexistence material. The main ingredients are the tetragonal and cubic phases. The tetragonal phase (TP) has a high performance, which could provide additional piezoelectric properties. The XRD of the PZT ceramic particles that 
were heated to various temperatures is shown as Fig. 6 . When the heat treatment temperature increased, a diffraction peak appeared at a $44^{\circ}$ diffraction angle. This diffraction peak was determined to be the characteristic peak of the TP. The diffraction peak reached the maximum intensity when the heat treatment temperature reached $1200{ }^{\circ} \mathrm{C}$. The relative contents of the tetragonal and cubic phases were calculated using the following equation:

$$
M_{T}=\frac{I_{T}(002)+I_{T}(200)}{I_{T}(002)+I_{T}(200)+I_{R}(200)} \times 100 \%,
$$

where $M_{T}$ is the volume content of the tetragonal phase, $I_{T}(002)$ and $I_{T}(200)$ are the intensities of the tetragonal phase's two characteristic peaks $T(002)$ and $T(200)$, and $I_{R}(200)$ is the intensity of the cubic phase's characteristic peak $R(200){ }^{(9)}$

The characteristic peak change of the PZT ceramic particles before and after heating is shown in Fig. 7. The solid line is the XRD patterns of the initial powders and the dotted line is the XRD patterns of the powders heated at $1200{ }^{\circ} \mathrm{C} . T(002)$ and $T(200)$ are the two diffraction peaks of the tetragonal phase and $R(200)$ is the diffraction peak of the cubic phase. When the temperature reached $1200{ }^{\circ} \mathrm{C}$, the intensities of $T(002)$ and $T(200)$ increased and the intensity of $R(200)$ decreased. Equation (1) was used to determine that when the heat treatment temperature reached $1200{ }^{\circ} \mathrm{C}$, the relative content of the tetragonal phase increased from 62 to $85 \%$. The heat treatment of the PZT ceramic particles improved the tetragonal phase content, which improved the piezoelectric activity of the piezoelectric ceramic particles.

\subsection{Thermogravimetry-differential scanning analysis (TG-DSC) of PZT ceramic particles}

The TG-DSC of PZT ceramic particles is shown in Fig. 8. When the temperature increased from $0-600{ }^{\circ} \mathrm{C}$, the PZT ceramic particles went through an endothermic process, and the weight of the particles decreased. The water and the low-molecular-weight organic matter adsorbed

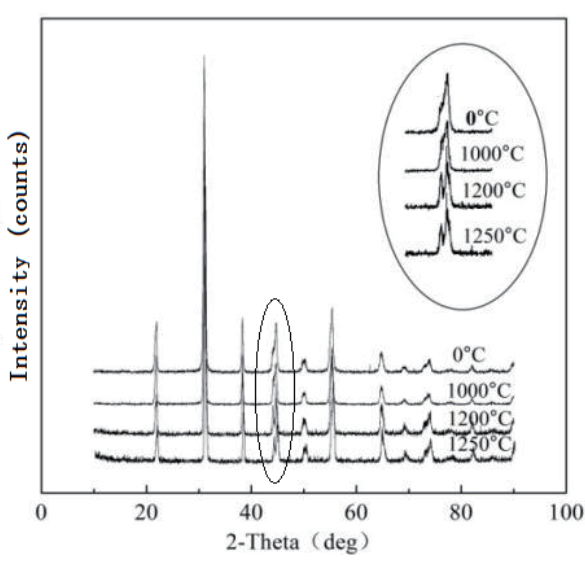

Fig. 6. XRD patterns of PZT ceramic particles heated to various temperatures.

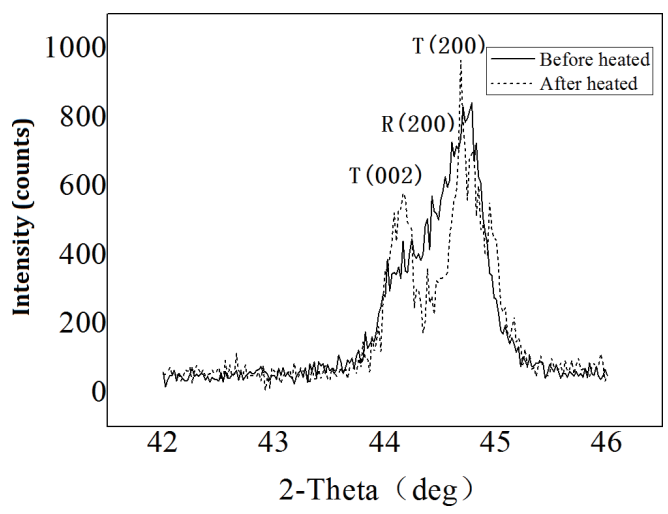

Fig. 7. Characteristic peak changes of PZT ceramic particles before and after heating. 


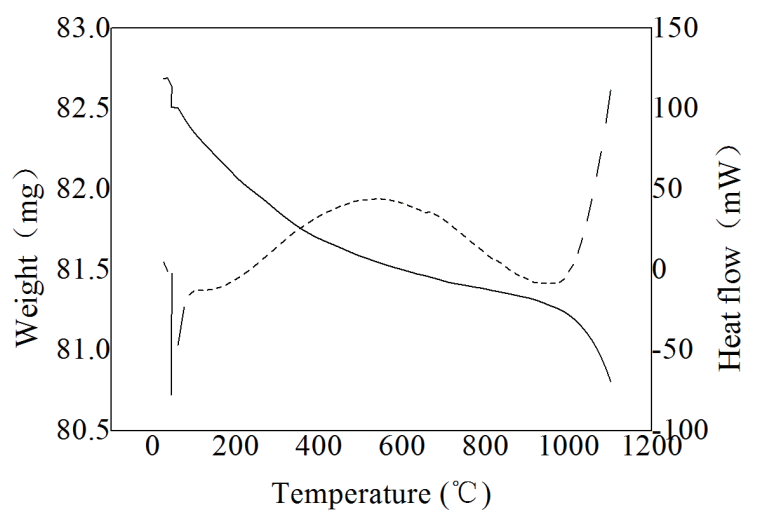

Fig. 8. TG-DSC of PZT ceramic particles.

onto the surface of the volatile particles or the breakdown resulted in the loss of weight. ${ }^{(10-12)}$ When the temperature increased from $600-1000{ }^{\circ} \mathrm{C}$, the PZT ceramic particles went through an exothermic process, and the weight of the particles decreased. ${ }^{(13)}$ The oxidative decomposition of the strong groups (such as $-\mathrm{OH},-\mathrm{C}=\mathrm{O}$, and $-\mathrm{COOH}$ ) adsorbed onto the surface of the particles results in the loss of weight. When the temperature was over $1000{ }^{\circ} \mathrm{C}$, the PZT ceramic particles went through an endothermic process, where the weight of the particles decreased. The volatility of the $\mathrm{PbO}$ and the phase change of the internal particles resulted in this phenomenon.

\section{Results and Discussion}

\subsection{SEM image of piezoelectric film}

The SEM image of the piezoelectric film made with the initial powders is shown in Fig. 9. The SEM image of the piezoelectric film made with the PZT powders heated at $1200{ }^{\circ} \mathrm{C}$ is shown in Fig. 10. These figures show that the piezoelectric ceramic particles were uniformly distributed in the polymer matrix. The system was a 0-3 type. The film made with the PZT powders heated at $1200{ }^{\circ} \mathrm{C}$ had superior interface compatibility, as well as less defects between the ceramic particles and the matrix. These results indicated that the heat treatment leads to the decomposition of the strong groups at the surface of the piezoelectric ceramic particles, the surface activity of the particles was reduced, and the interface compatibility of the piezoelectric ceramic particles and the polymer matrix was enhanced.

\subsection{Conductivity of piezoelectric film}

Figure 11 shows the conductivity of the piezoelectric film at room temperature and $1 \mathrm{kHz}$. The conductivity of the piezoelectric film slowly increased when the temperature was increased. When the heat treatment temperature reached $1200{ }^{\circ} \mathrm{C}$, the conductivity of the piezoelectric 


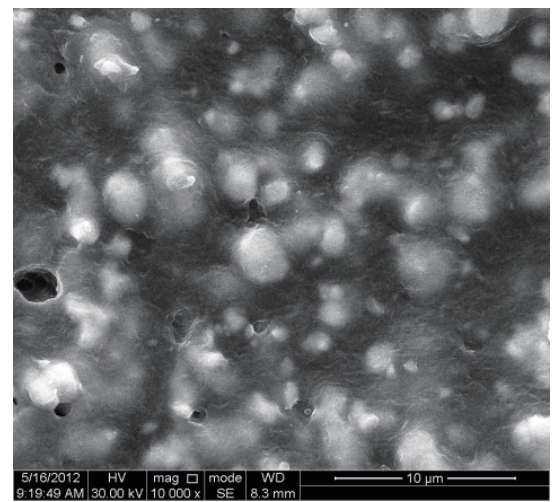

(a)

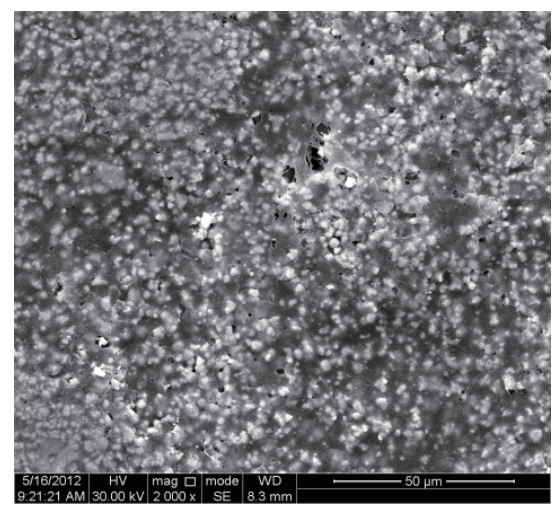

(b)

Fig. 9. Piezoelectric film made with initial powders. (a) $\times 10000$ and (b) $\times 2000$.

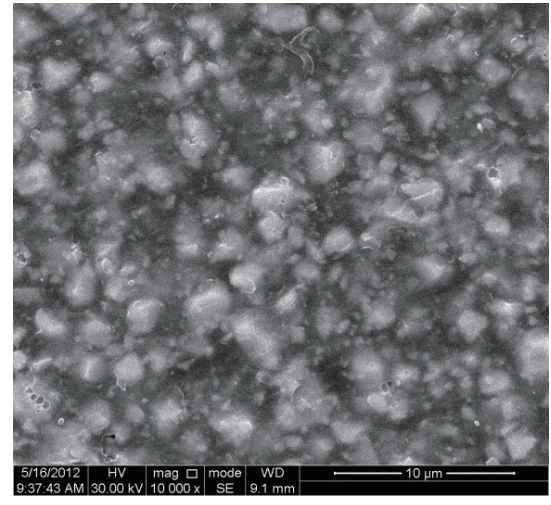

(a)

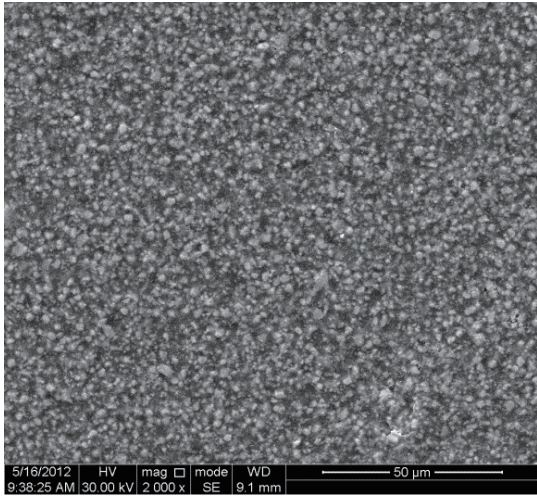

(b)

Fig. 10. Piezoelectric film made with PZT powders heated at $1200^{\circ} \mathrm{C}$. (a) $\times 10000$ and (b) $\times 2000$.

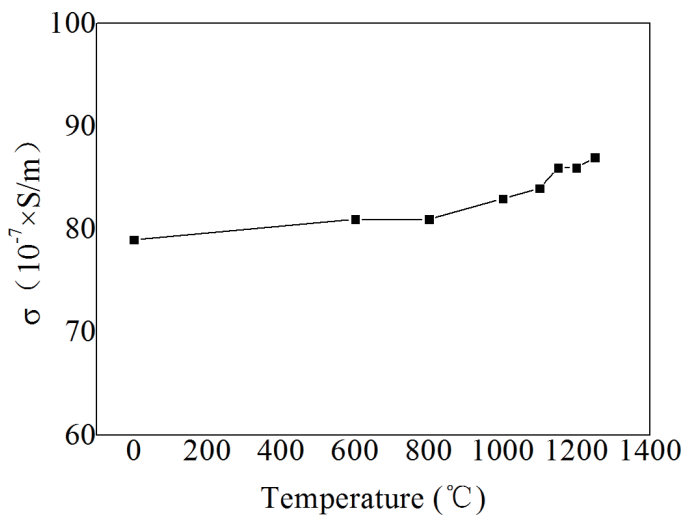

Fig. 11. Conductivity of piezoelectric film influenced by heat treatment temperature.

film was $86 \times 10^{-7} \mathrm{~S} / \mathrm{m}$, which was 1.09 times higher than that of the untreated film. This result indicated that the heat treatment increased the conductivity of the piezoelectric film, but the effect was small. 


\subsection{Dielectric properties of piezoelectric film}

Figure 12 shows the dielectric constant and dielectric loss of the piezoelectric film influenced by the heat treatment temperature at room temperature and $1 \mathrm{kHz}$. The dielectric constant of the piezoelectric film slowly increased as the temperature increased. The dielectric loss of the piezoelectric film slowly decreased as the temperature increased. When the heat treatment temperature reached $1200^{\circ} \mathrm{C}$, the dielectric constant of the piezoelectric film reached 106, which increased by approximately $10 \%$. The dielectric loss of the piezoelectric film reached $84 \mathrm{mU}$, which was a $9 \%$ decrease. These results indicated that the heat treatment could improve the dielectric properties of the piezoelectric film.

\subsection{Piezoelectric properties of piezoelectric film}

Figure 13 shows the $d_{33}$ and $g_{33}$ of the piezoelectric film, which were influenced by the heat treatment temperature. When the temperature was below $1000^{\circ} \mathrm{C}$, the $d_{33}$ of the piezoelectric film slowly increased as the temperature increased. When the temperature was over $1000{ }^{\circ} \mathrm{C}$, the $d_{33}$ increased rapidly as the heat treatment temperature increased. When the heat treatment temperature was over $1200{ }^{\circ} \mathrm{C}$, the $d_{33}$ decreased as the temperature increased. The $\mathrm{PbO}$ of piezoelectric ceramic particles was volatile when the heat treatment temperature was over 1200 ${ }^{\circ} \mathrm{C}$. The internal structure of the particles changed, which led to the decrease in piezoelectric activity. When the heat treatment temperature was $1200{ }^{\circ} \mathrm{C}$, the $d_{33}$ of the piezoelectric film reached $45 \mathrm{pC} / \mathrm{N}$, which was 1.8 times higher than that of the untreated film. The $g_{33}$ of the film was $44 \mathrm{mV} \cdot \mathrm{m} / \mathrm{N}$, which was 1.4 times higher than that of the untreated film. The change rules of the piezoelectric constant verified the conclusions obtained in Sects. 3.2 and 3.3. A reasonable heat treatment temperature could improve the tetragonal phase content, which could increase the piezoelectric activity.

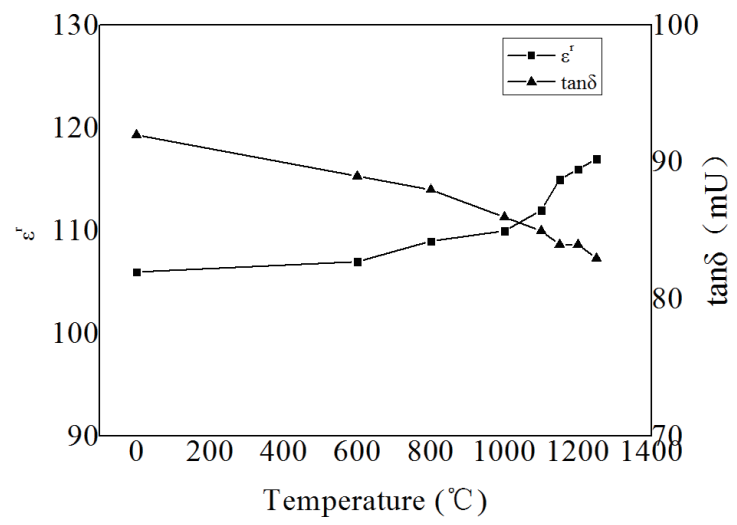

Fig. 12. Dielectric properties of piezoelectric film influenced by heat treatment temperature. 


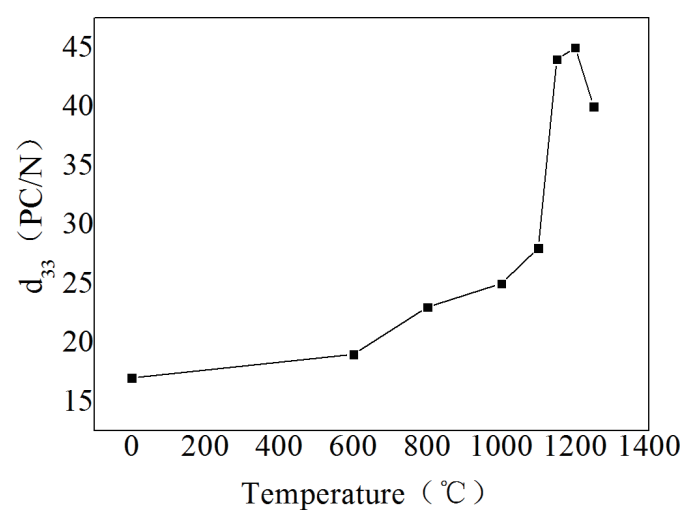

(a)

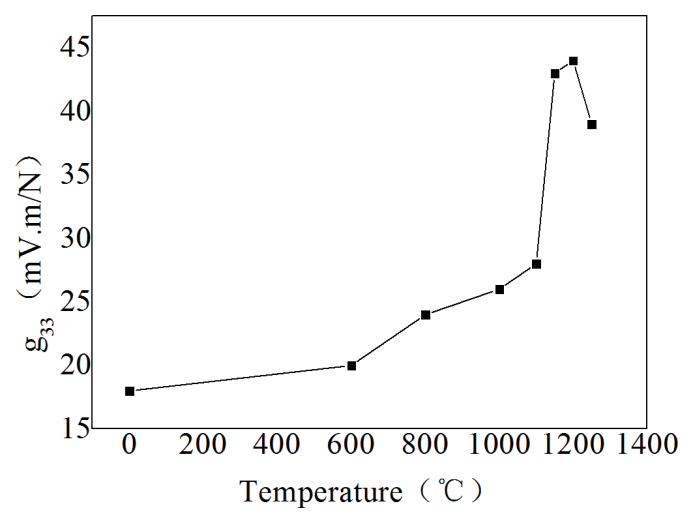

(b)

Fig. 13. (a) Piezoelectric strain constant and (b) piezoelectric voltage constant of piezoelectric film.

\subsection{Electromechanical coupling coefficients of piezoelectric film}

Figure 14 shows the $K_{p}$ and $K_{t}$ of the piezoelectric film, which were influenced by the heat treatment temperature. The $K_{p}$ and $K_{t}$ of the piezoelectric film slowly increased as the temperature increased. When the temperature was below $1000^{\circ} \mathrm{C}$, the $K_{p}$ and $K_{t}$ increased as the temperature increased. When the heat treatment temperature was greater than $1200{ }^{\circ} \mathrm{C}$, the $K_{p}$ and $K_{t}$ decreased as the temperature increased. When the heat treatment temperature was $1200{ }^{\circ} \mathrm{C}$, the $K_{p}$ of the piezoelectric film reached 0.212 , which was 1.45 times higher than that of the untreated film. The $K_{t}$ of the film was 0.208 , which was 1.44 times higher than that of the untreated film. The change rules of the electromechanical coupling coefficients verified the conclusions obtained in Sect. 4.4.

\subsection{Mechanical quality factor of piezoelectric film}

Figure 15 shows the $Q_{m}$ of the piezoelectric film, which was influenced by the heat treatment temperature. When the heat treatment temperature was below $1000{ }^{\circ} \mathrm{C}$, the $Q_{m}$ of the piezoelectric film slowly decreased as the temperature increased. When the heat treatment temperature increased from 1000 to $1200{ }^{\circ} \mathrm{C}$, the $Q_{m}$ decreased rapidly as the heat treatment temperature increased. When the heat treatment temperature was over $1200^{\circ} \mathrm{C}$, the $Q_{m}$ increased as the temperature increased. When the heat treatment temperature was $1200{ }^{\circ} \mathrm{C}$, the $Q_{m}$ of the piezoelectric film reached 0.349 , which was 1.45 times higher than that of the untreated film. The $K_{t}$ of the film was 0.208 , which decreased $30 \%$ more than that of the untreated film.

\section{Results of Cantilever Beam Experiment}

In this experiment, the cantilever beam was vibrated by 1 and $20 \mathrm{~Hz}$ sinusoidal loads. The responses of the piezoelectric film sensors and strain gauge sensors under the sinusoidal load are shown in Fig. 16. The response of the strain gauge sensors was associated with the voltage 


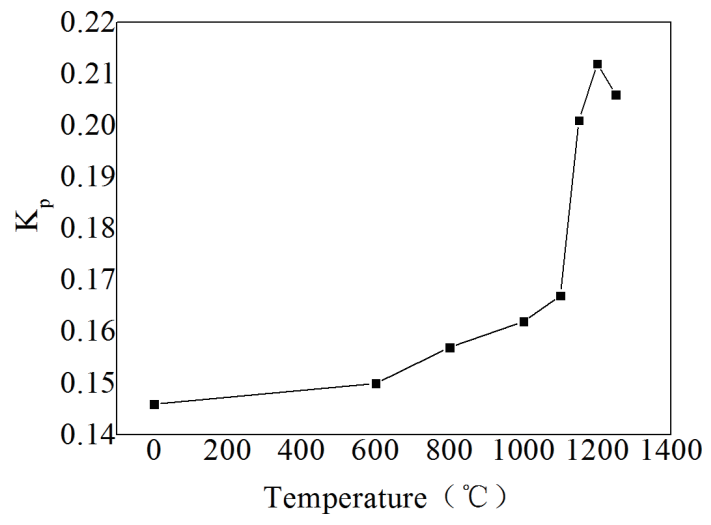

(a)

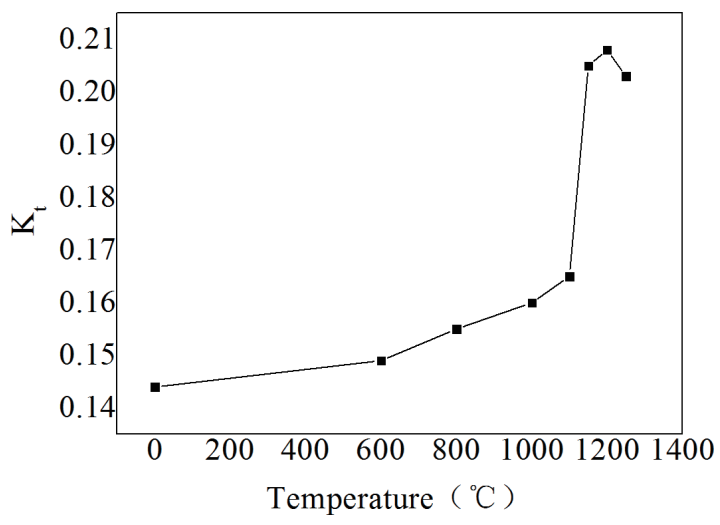

(b)

Fig. 14. Electromechanical coupling coefficients of piezoelectric film. (a) $K_{p}$ and (b) $K_{t}$.

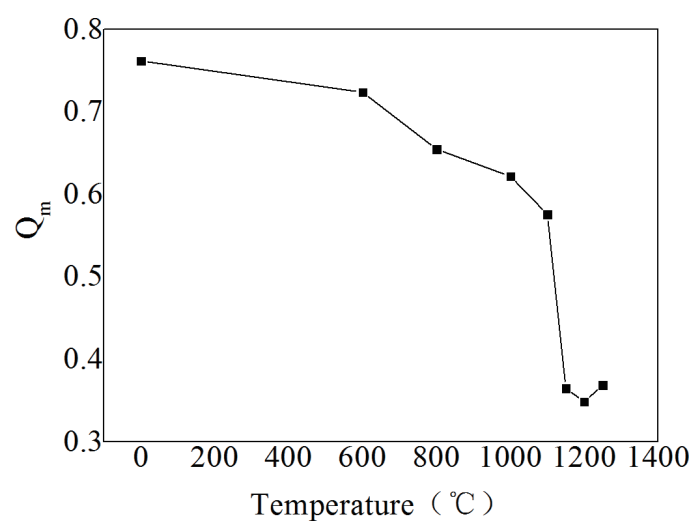

Fig. 15. Mechanical quality factors of piezoelectric film.

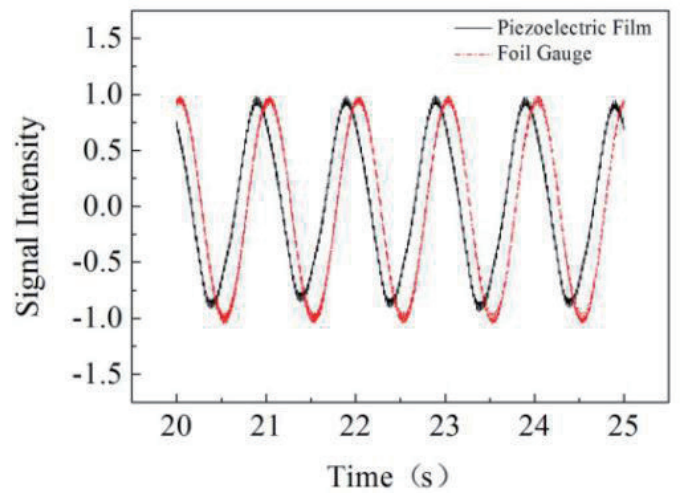

(a)

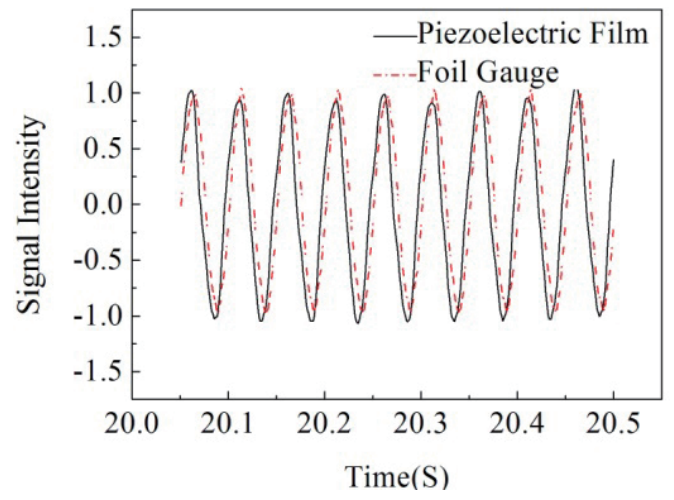

(b)

Fig. 16. (Color online) Responses of piezoelectric film sensors and strain gauge sensors. (a) 1 and (b) $20 \mathrm{~Hz}$.

value of the external power, so the signals seen in Fig. 16 were homogenized. The $Y$-axis of Fig. 16 was the measured value divided by the maximum measured value of each data set.

The responses of the piezoelectric film sensors and strain gauge sensors under a sinusoidal load are denoted as the standard sine curve in Fig. 16. These results indicated that under a low 
frequency load, the piezoelectric film sensors and strain gauge sensors correctly reflected the deformation of the structure. The signals of the strain gauge sensors were slightly delayed from the other two sensors. This showed that the synchronicity and sensitivity of the piezoelectric film sensors were higher than those of the strain gauge sensors. The piezoelectric film sensors have value in both research and application.

\section{Conclusions}

The effect that the heat treatment had on the performance of the piezoelectric film was examined. The analysis and comparisons resulted in the following conclusions:

- The heat treatment of the PZT ceramic particle improved the content of the tetragonal phase, which improved the piezoelectric activity of the piezoelectric ceramic particles.

- The heat treatment resulted in the decomposition of strong groups at the piezoelectric ceramic particle surface, the surface activity of the particles decreased, and the interface compatibility of the piezoelectric ceramic particles and the polymer matrix was enhanced.

- The enhanced interface compatibility will lead to defects between the ceramic particles decreased; the polarization voltage was increased, and finally, the piezoelectric properties were improved.

- The heat treatment improved the piezoelectric activity of the piezoelectric film. The reasonable heat treatment temperature was $1200^{\circ} \mathrm{C}$.

- The synchronicity and sensitivity of the piezoelectric film sensors were higher than those of the strain gauge sensors. Piezoelectric film sensors have great value in both research and application. The piezoelectric composite film sensors were effective for use in the real-time monitoring of structure stress.

\section{Acknowledgments}

This research was financially supported by the National Natural Science Foundation of China under Grant No. U1334209, Key Laboratory of Hebei University of Architecture, Science and Technology of Hebei Province under Grant No. 15211112, and the Education Department of Hebei Province under Grant Nos. QN2014169 and QN2016066.

\section{References}

1 Y. J. Lmai, D. Tadaki, and T. Ma: Sens. Actuators, B 247 (2017) 479.

2 M. Reusch, K. Holc, and L. Kirste: Procedia Eng. 168 (2016) 537.

3 S. Yoshida, H. Hanzawa, and K. Wasa: Sens. Actuators, A 239 (2016) 201.

4 S. M. Hosseini and A. A. Yousefi: Org. Electron. 50 (2017) 121.

5 M. V. Silibin, V. S. Bystrov, and D. V. Karpinsky: Appl. Surf. Sci. 421 (2017) 42.

6 K. H. Baumgärtel, D. Zöllner, and K. L. Krieger: Procedia Technol. 26 (2016) 491.

7 J. Dargahi, W. F. Xie, and P. Ji: Mechatronics 27 (2008) 1.

8 S. C. Choi, J. S. Park, and H. Kim: Compos. Struct. 76 (2006) 362.

9 S. Hurlebaus, U. Stobener, and L. Gaul: Comput. Struct. 86 (2008) 251.

10 S. G. Gong, A. Safari, and S. J. Jang: Ferroelectric Lett. 5 (1986) 131.

11 S. G. Gong, A. Safari, and R. E. Newnham: Proc. Sixth Ferroelectrics 41 (1986) 281.

12 W. K. Sakamoto, F. P. Marin, and D. K. Gupta: Sens. Actuators, A 100 (2002) 165.

13 L. Wang and Z. M. Dang: Appl. Phys. Lett. 87 (2005) 42. 


\section{About the Authors}

Yudong Zhang received his master's degree in 2012 from Harbin Institute of Technology (HIT), P.R. China. Currently, he is an assistant professor in Hebei Institute of Architecture and Civil Engineering. His research focus is mainly on smart structures and materials.

Jin Guo received his Ph.D. degree in 2014 from Tongji University. Currently, he is an assistant professor at Shijiazhuang Tiedao University.

Yan Han received her master's degree in 2008 from Jiangsu University. Currently, she is an assistant professor in Hebei Institute of Architecture and Civil Engineering.

Jing Mi received his bachrlor's degree in 2013 from Hebei Institute of Architecture and Civil Engineering. Currently, he is a staff member in Construction Bureau of Xuan Hua. 